(C) 2007 IEEE. Reprinted, with permission, from Youguang Guo, A Direct Torque Controlled Surface Mounted PMSM Drive with Initial Rotor Position Estimation Based on Structural and Saturation Saliencies, Industry Applications Conference, 2007. 42nd IAS Annual Meeting. Conference Record of the 2007 IEEE, Sept. 2007. This material is posted here with permission of the IEEE. Such permission of the IEEE does not in any way imply IEEE endorsement of any of the University of Technology, Sydney's products or services. Internal or personal use of this material is permitted. However, permission to reprint/republish this material for advertising or promotional purposes or for creating new collective works for resale or redistribution must be obtained from the IEEE by writing to pubspermissions@ieee.org. By choosing to view this document, you agree to all provisions of the copyright laws protecting it 


\title{
A Direct Torque Controlled Surface Mounted PMSM Drive with Initial Rotor Position Estimation Based on Structural and Saturation Saliencies
}

\author{
Ying Yan, Jianguo Zhu, and Youguang Guo \\ Faculty of Engineering \\ University of Technology, Sydney \\ PO Box 123, Broadway, NSW 2007, Australia \\ yingyan@eng.uts.edu.au, joe@eng.uts.edu.au, youguang@eng.uts.edu.au
}

\begin{abstract}
For a practical direct torque controlled (DTC) permanent magnet synchronous motor (PMSM) drive system, the information of the initial rotor position, which is usually obtained by a mechanical position sensor, is essential for starting under the full load. In order to avoid the disadvantages of using mechanical position sensors, great efforts have been made on the development of sensorless control schemes. This paper presents an initial rotor position estimation scheme for a DTC PMSM drive based on a nonlinear model of PMSM incorporating both structural and saturation saliencies. Firstly, the performance of the estimation scheme is simulated within Matlab/Simulink environment. Based on the simulation results, the estimation scheme has been investigated. Experiments have also been carried out to identify the initial rotor position of a surface mounted PMSM (SPMSM). In the experiment, specially designed high voltage pulses are applied to amplify the saturation saliencies. The peak currents corresponding to the voltage pulses are used, in combination with the inductance patterns, to determine the $d$-axis position and the polarity of the rotor. Finally, the presented initial rotor position identification strategy has been implemented in a sensorless DTC drive for an SPMSM. Experiments are conducted to confirm the effectiveness of the method and the performance of the drive system.
\end{abstract}

Keywords-Permanent magnet sysnchonous motor (PMSM) drive; surfaced mounted PMSM; nonlinear model of PMSM; structural saliency; saturation saliency; inductance; direct torque control; initial rotor position estimation; voltage pulse.

\section{INTRODUCTION}

For a permanent magnet synchronous motor (PMSM) drive, the rotor position information, which is usually detected by using a mechanical position sensor, is essential for the inverter commutation to control the frequency and position of the stator current vectors so that the motor can be started under full load. In order to avoid the disadvantages of using position sensors, such as higher system cost and lower system reliability, sensorless rotor position estimation schemes have become more desirable [1-3]. Among the modern control strategies, the direct torque control (DTC) scheme is preferred because of its simplicity and fast torque response. Moreover, in DTC scheme, sensorless control strategy can be realized at high speeds. The only requirement is to acquire the initial rotor position information for starting the motor. Therefore, an accurate identification of the initial rotor position is critical for the DTC PMSM drive.

Most of the present initial rotor position estimation schemes utilize the structural and magnetic saturation saliencies which exist in all types of PMSMs. In a PMSM, the structural and magnetic saturation saliencies could be reflected in the nonlinear winding inductances which are functions of the rotor position and stator currents. The inductances in several space phasor directions were measured and compared to estimate the rotor position in [1]. Most of the present methods can readily estimate the initial rotor position of motors with large structural saliency, e.g. the interior PMSM. However, it becomes difficult to detect the initial rotor position of a surface mounted PMSM (SPMSM). In [2], the saturation saliency related inductance variation in an SPMSM was investigated. In [3], high voltage pulses were applied to amplify the effect of saturation saliency of the stator core, and the amplitude difference of the three phase current responses was used to estimate the $d$-axis and the polarity of the rotor at the same time. This method is further investigated in this paper.

This paper firstly presents a nonlinear model of PMSMs with both structural and magnetic saturation saliencies to enable the numerical simulation of new rotor position detection algorithms. In this model, the nonlinear differential inductances of the phase windings are measured and expressed by Fourier series as functions of stator currents and rotor positions. Using this model, we investigate the initial rotor position estimation scheme proposed in [5] by numerical simulation. It is found that the high voltage pulses are effective for identifying the saturation saliency of SPMSMs. The initial rotor position estimation schemes can be realized by simulation. Based on the simulation results and the methods in [3], the scheme for identifying the initial rotor position and polarity of SPMSM is developed. The scheme is confirmed by experimental results. Finally, the developed initial rotor position identification scheme is incorporated in a sensorless DTC drive for an SPMSM. Experiments are conducted to verify the performance of the drive system. 


\section{NONLINEAR MODEL OF PMSM WITH SALIENCIES}

In a PMSM, the total magnetic field is produced by the rotor magnets and stator currents. Although the $B-H$ characteristic of the magnetic core is nonlinear, the magnetic circuit can be considered as piecewisely linearized around the operating point at a given rotor position. For phase $a$, the total flux linkage, $\psi_{a}$, can be separated into two components as $\psi_{a s}$ and $\psi_{a f}$ caused by the stator current $i_{m s}$, and rotor magnets, respectively [4-5]. The flux linkage $\psi_{a s}$ can be further separated into components due to individual stator phase currents as $\psi_{a a}$, $\psi_{a b}$ and $\psi_{a c}$, which are the functions of $i_{a}, i_{b}, i_{c}, i_{f}$ and rotor position, $\theta_{r}$. By analyzing the magnetic circuit of the PMSM, the state variable equations can be obtained as:

$$
v_{j}=R_{j} i_{j}+\sum_{k=a}^{c} L_{j k}^{\prime} \frac{d i_{k}}{d t}+e_{j f}+e_{j \theta}(j=a, b, c)
$$

where $v_{j}$ are the terminal voltages, $R_{j}$ the winding resistances, and $i_{j}$ the currents of phases $a, b$ and $c$, respectively. The inductances in (1) are the self and mutual incremental inductances. $e_{j f}=\omega_{r} d \lambda_{j f} / d \theta_{r}$, are the induced emfs, $e_{j \theta}=\omega_{r}\left(\partial \psi_{j \alpha} / \partial \theta_{r}\right.$ $\left.+\partial \psi_{j b} / \partial \theta_{r}+\partial \psi_{j c} / \partial \theta_{r}\right)$ the emfs induced by the variation of flux linkage due to the saliencies, and $\omega_{r}=d \theta_{r} / d t$ is the mechanical angular speed of the rotor.

The electromagnetic torque of the PMSM can be calculated by taking the partial derivative of the system co-energy with respect to the rotor angle as:

$$
\begin{aligned}
T_{e m}= & i_{a} \frac{\partial \psi_{a f}}{\partial \theta_{r}}+i_{b} \frac{\partial \psi_{b f}}{\partial \theta_{r}}+i_{c} \frac{\partial \psi_{c f}}{\partial \theta_{r}}+\frac{i_{a}^{2}}{2} \frac{\partial L_{a a}}{\partial \theta_{r}}+\frac{i_{b}^{2}}{2} \frac{\partial L_{b b}}{\partial \theta_{r}}+\frac{i_{c}^{2}}{2} \frac{\partial L_{c c}}{\partial \theta_{r}} \\
& +\frac{\partial L_{a b}}{\partial \theta_{r}} i_{a} i_{b}+\frac{\partial L_{a c}}{\partial \theta_{r}} i_{a} i_{c}+\frac{\partial L_{b c}}{\partial \theta_{r}} i_{c} i_{b}
\end{aligned}
$$

where the inductances are apparent self and mutual inductances. There are two components of the electromagnetic torque. One is produced by the stator currents and rotor magnets, and the other is caused by the saliencies.

\section{DETERMINATION OF NONLINEAR INDUCTANCE}

In order to measure the self and mutual inductances with structural and saturation saliencies, which could be used in the presented model, a special testing method is designed [4-5]. By this method, the inductances at different rotor positions have been measured. Meanwhile, DC offsets with different amplitudes and positions, $i_{D C}$, are used to emulate the saturation effect of the three phase currents, which correspond to the saturation saliency.

The experiment is carried out on a 6 pole SPMSM with a locked rotor. On one hand, different DC offsets are injected to one of the stator windings to emulate the effect of stator fluxes. On the other hand, a small AC current is applied to one phase while the other two are open-circuited. The power, voltage and current of the excited phase are measured and the voltages of the other two phases are also recorded by a power analyzer. The self and mutual inductances of phases $a, b$ and $c$ at different rotor positions and stator fluxes can then be worked out via circuit analysis. The Fourier series is utilized to express the phase inductances. Due to the nonlinear characteristics of the magnetic core, the inductances vary with stator currents. Therefore, the inductances can be considered as a periodic function of the rotor angular positions and varies nonlinearly with the stator currents, due to the saturation saliency. Consequently, different sets of coefficients $a_{0}, a_{m}$, and $b_{m}(m=1$, $2, \ldots, n)$ can be determined at different currents, and expressed as the functions of the corresponding current. The following Fourier series with current dependent polynomial coefficients appears to be the most appropriate:

$$
\begin{aligned}
& L\left(i_{D C}, \theta_{r}, \theta_{D C}\right)=a_{0}\left(i_{D C}, \theta_{D C}\right) \\
& +\sum_{m=1}^{n}\left(a_{m}\left(i_{D C}, \theta_{D C}\right) \cos \left(m \theta_{r}\right)+b_{m}\left(i_{D C}, \theta_{D C}\right) \sin \left(m \theta_{r}\right)\right)
\end{aligned}
$$

The number of terms of the Fourier series, $n$, can be chosen with sufficient accuracy through the nonlinear curve fitting of the measured phase inductances, $L\left(i_{D C}, \theta_{r}, \theta_{D C}\right)$, where $i_{D C}$ and $\theta_{D C}$ refer to the various amplitudes and positions of the applied DC offset, respectively, and $\theta_{r}$ is the rotor position where the experiment is carried out.

As discussed above, the stator winding inductances are defined and measured in the $a b c$ reference frame. In order to simplify the inductance expressions and unify the definitions of $\theta_{s}$ and $\theta_{r}$, the angle between the stator flux, $\Phi_{s}$, and the stator winding axis of phase $a$, denoted by $\alpha$, and the angle between the stator and rotor fluxes, denoted by $\delta$, are adopted in the inductance expressions instead of $\theta_{s}$ and $\theta_{r}$. The amplitude of $\Phi_{s}$ is determined by $i_{D C}$ in the experiment. In the PMSM, the the magnetization component of the stator currents, $i_{m s}$, has been used to represent the amplitude of the stator flux, $\left|\Phi_{s}\right|$, which corresponds to the various $i_{D C}$ in the test. With the new definition, the inductance can be expressed as $L\left(i_{m s}, \delta, \alpha\right)$ :

$$
\begin{aligned}
& L\left(i_{m s}, \delta, \alpha\right)=a_{0}\left(i_{m s}, \alpha\right) \\
& +\sum_{m=1}^{n}\left(a_{m}\left(i_{m s}, \alpha\right) \cos (m \delta)+b_{m}\left(i_{m s}, \alpha\right) \sin (m \delta)\right)
\end{aligned}
$$

According to the experimental results, the inductances at $\alpha=0^{\circ}, 120^{\circ}$ and $240^{\circ}$ for arbitrary $\delta$ and $i_{m s}$ can be obtained by curve fitting of the experimental inductances using (4). Since the inductances at three positions of the stator flux, various stator flux amplitudes and rotor positions have been obtained, interpolation can be applied to estimate the inductance values for any fixed $\alpha, \delta$ and $i_{m s}$. Due to the symmetry of the three phase stator windings, the self inductances of phases $b$ and $c$ at arbitrary rotor position and stator current can be identified and estimated in a similar way to the phase $a$ self inductance. In general, the inductances for arbitrary rotor and stator fluxes could be obtained with the procedure in Fig. 1.

With (4), the self and mutual inductances at certain rotor position and stator fluxes are obtained. Fig. 2(a) shows $L_{c c}$ at different $\alpha$ with $\delta=0^{\circ}-360^{\circ}$ and $i_{m s}=5.5 \mathrm{~A}$. The inductance estimation procedure can also be used to acquire the mutual inductance expression. Fig. 2(b) shows the mutual inductance 
between phases $a$ and $b, L_{a b}$, with a DC offset of $5.5 \mathrm{~A}$ at different $\alpha$. In this way, the three phase self and mutual inductances under any operation conditions can be obtained based on the experimental results and (4). The structural and saturation saliencies of the motor magnetic circuit could be mapped out in terms of the three phase self and mutual inductances. Finally, the nonlinear expression of the self and mutual inductances at arbitrary rotor position and stator flux can be readily incorporated into the PMSM model.

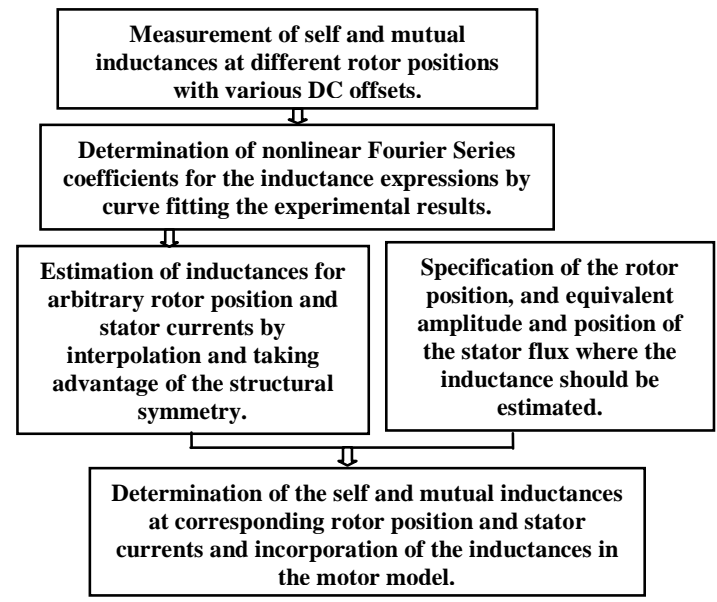

Figure 1. Flow chart to identify inductances with saliencies.

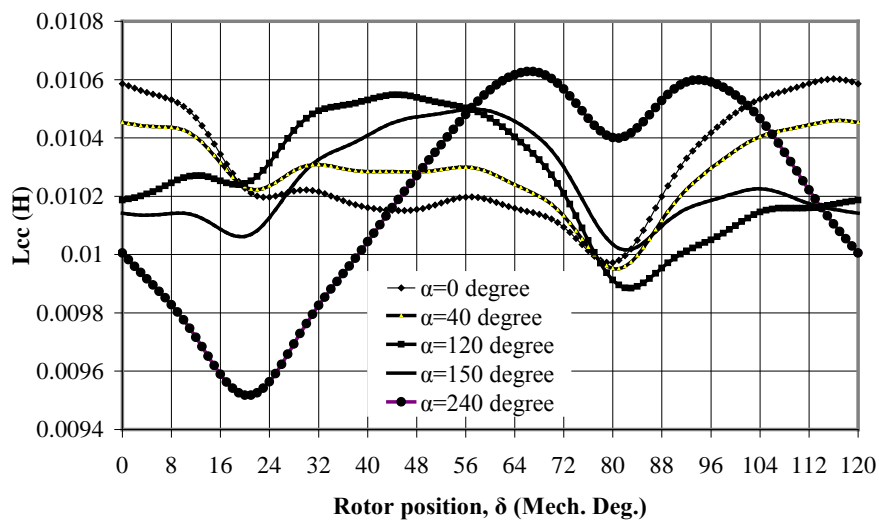

(a)

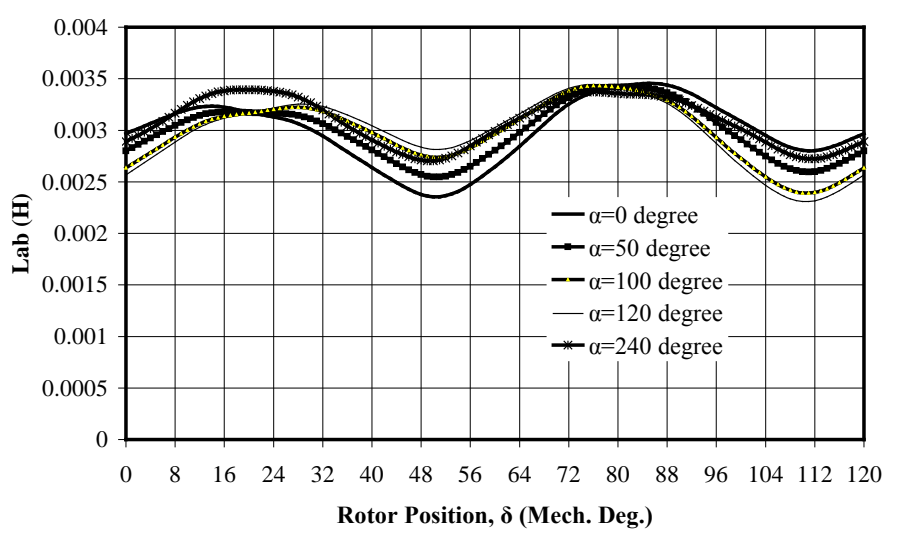

(b)

Figure 2. Inductances with a DC offset of $5.5 \mathrm{~A}$ : (a) $L_{c c}$ and (b) $L_{a b}$.

\section{SimUlATION OF INITIAL ROTOR POSITION ESTIMATION}

\section{A. Estimation Principle}

It is possible to detect when the stator winding is aligned with the rotor magnets by measuring the self and mutual inductances of three phase windings [3]. Fig. 2 depicts the winding inductance as a function of rotor positions and stator currents when the stator flux is superimposed on the flux produced by the rotor PMs. It can be inferred that when the north pole is aligned with the axis of the phase $a$ winding, the DC current in phase $a$ causes the magnetic core to become more saturated, and the inductances slightly decrease compared with those under the situation without stator current. On the contrary, when the south pole is aligned with the stator winding, the inductances slightly increase. The difference of the winding inductances for the north and south poles can be employed to distinguish the polarity of the rotor pole.

By choosing an appropriate voltage pulse width and applying it to each phase winding, the stator currents partially saturate the stator iron, enabling the algorithm to discern between a north pole and a south pole, and subsequently, the absolute position.

\section{B. Simulated Peak Currents}

A Simulink model of the PMSM at standstill has been built and the voltage pulse injection algorithm is simulated with the measured parameters of the test SPMSM [4-5].

The fundamental procedure of this scheme is summarized as follows. At an unknown initial rotor position, a positive pulse is applied to one of the three phase terminals for a certain duty, while the terminals of the other two phases are applied with negative pulses. With this positive pulse, the positive peak current value is acquired. After that, the positive pulse is followed by a negative pulse and the negative pulses by positive pulses to bring the phase currents back to zero. Then, at the same rotor position, a negative pulse is applied to the same phase for the same duty, while the terminals of the other two phases are supplied with positive pulses. In this way, the negative peak current value is measured. After that, one positive pulse and two negative pulses are used to bring the phase currents back to zero. For example, the gate signals of 010 are created to inject a positive pulse to phase $b$ for a short period, and then immediately the gate signals of 101 are injected. After the stator currents become zero, the gate signals of 101 are created, and then immediately the gate signals of 010 are produced to drive the current in the phase winding to zero. The high voltage pulses are injected to the Simulink model, as depicted in Fig. 3, and the corresponding current responses are recorded, as illustrated in Fig. 4.

The high voltage pulses are applied to the three phase stator windings, respectively. The continuous inductance patterns based on the measured self and mutual inductances with DC offset are utilized as the parameters of the model. The simulated three phase peak currents within an electrical cycle at different rotor positions are illustrated in Fig. 5(a). By comparing the peak current waveforms under the nonsaturated conditions, it can be seen that the effect of the saturation saliency becomes sufficiently evident in the profiles 
of the peak currents with high voltage pulses. Similar experiments are carried out with the negative voltage pulses. The measured three phase peak currents within an electrical cycle at different rotor positions are shown in Fig. 5(b).

As shown in Fig. 5, the peak current distribution can be approximately regarded as sine waves with different frequencies versus the rotor position and modeled by: $I_{a p}=\Delta I_{0}+\Delta I_{01} \cos \theta+\Delta I_{02} \cos 2 \theta+\Delta I_{03} \cos 3 \theta+\Delta I_{04} \cos 4 \theta, \quad I_{b p}=\Delta I_{0}+$ $\Delta I_{01} \cos (\theta+2 \pi / 3)+\Delta I_{02} \cos (2 \theta-2 \pi / 3)+\Delta I_{03} \cos (3 \theta)+\Delta I_{04} \cos (4 \theta-$ $2 \pi / 3)$, and $I_{c p}=\Delta I_{0}+\Delta I_{01} \cos (\theta-2 \pi / 3)+\Delta I_{02} \cos (2 \theta+2 \pi / 3)$ $+\Delta I_{03} \cos (3 \theta)+\Delta I_{04} \cos (4 \theta+2 \pi / 3)$. The fitted positive and negative peak currents have also been plotted in the figure with the above model.

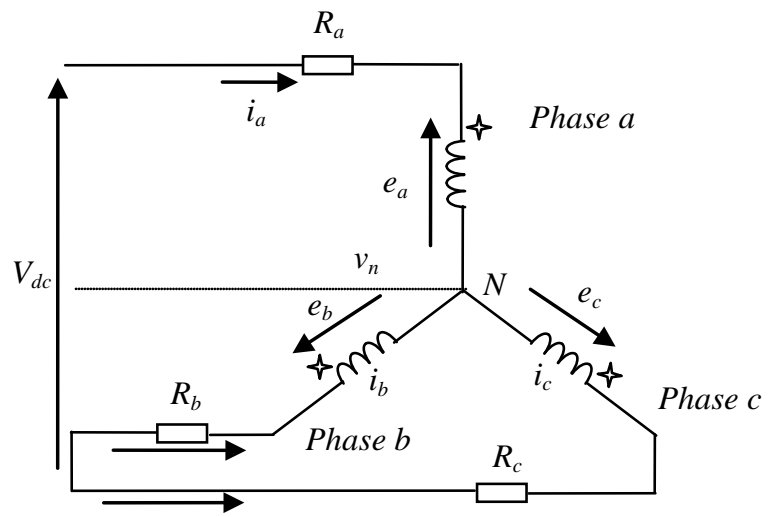

Figure 3. Equivalent circuit of the PM synchronous motor at standstill.

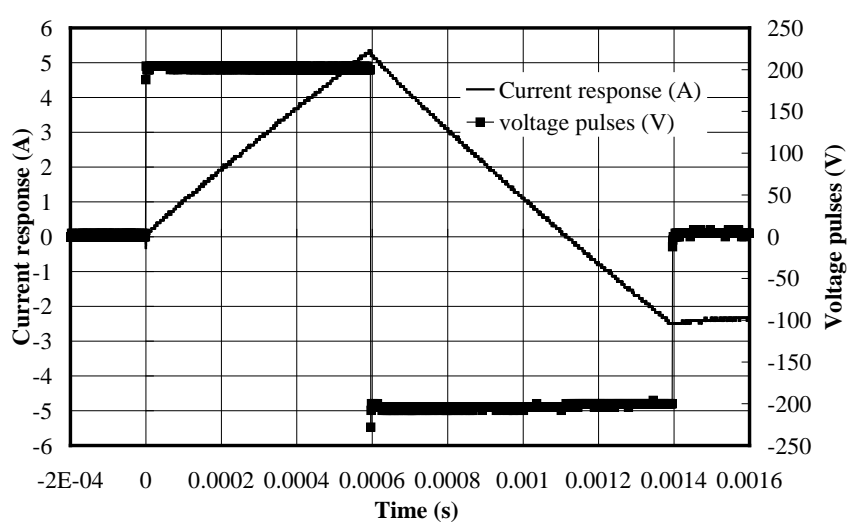

Figure 4. Simulated high voltage pulse and current response.

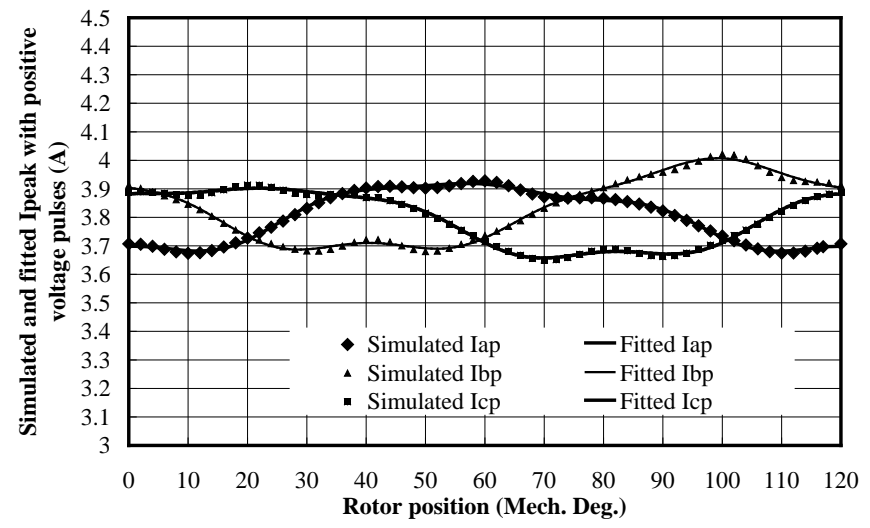

(a)

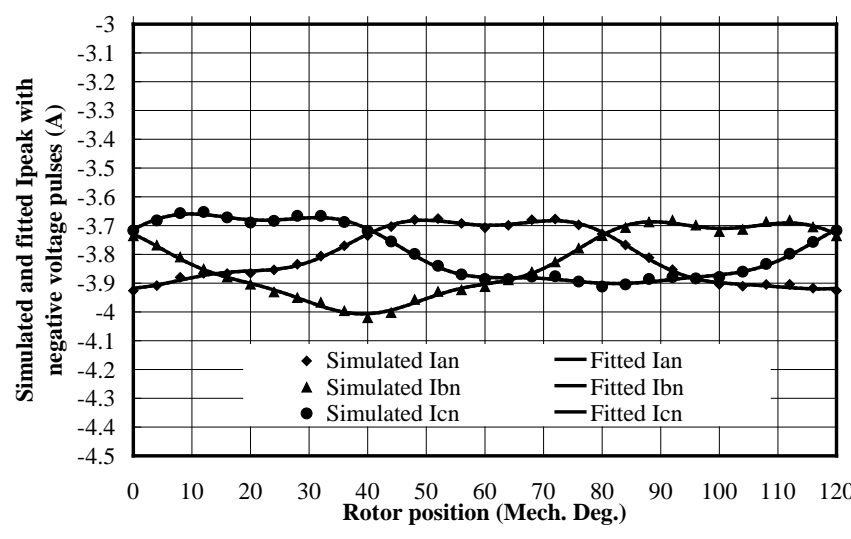

(b)

Figure 5. Curve fitting of the three phase peak currents due to high voltage pulses: (a) positive pulses and (b) negative pulses.

\section{Estimation Procedure and Results}

For the control of PMSM drives, the rotor electrical angular position information within a pole pair should be estimated. The initial rotor position is at the axis of the phase $a$ stator winding.

An $m$-file program is designed within the Matlab environment to realise the proposed algorithm. The simulated peak current curves of phases $a, b$ and $c$ at different rotor positions are stored in the form of a look up table. When the rotor is standstill, the gate signals of 100, 010, and 001 are sent to the PMSM and inverter models in the Matlab/Sinmulink environment to apply high voltage pulses to the three phase stator winding terminals, and the peak phase currents are recorded. Possible rotor positions can be readily found using a sectional interpolation method. For phase $a$, the possible rotor positions corresponding to the simulated peak current $I_{a p}$ can be acquired as $\theta_{a 1} \theta_{a 2}, \theta_{a 3}$ and so on, as plotted in Fig. 6(a). Similarly, for phases $b$ and $c$, the possible rotor positions corresponding to the simulated $I_{b p}$ and $I_{c p}$ can be obtained as $\theta_{b 1}$ $\theta_{b 2}, \theta_{b 3}, \theta_{c 1} \theta_{c 2}, \theta_{c 3}$ and so on, as illustrated in Fig. 6(b-c). Among all these possible rotor positions, the actual rotor position is given by the common angle of the all three phases. Fig. 7 compares the estimated rotor positions obtained by numerical simulation against the actual rotor positions. It can be inferred that this algorithm can produce satisfactory results.

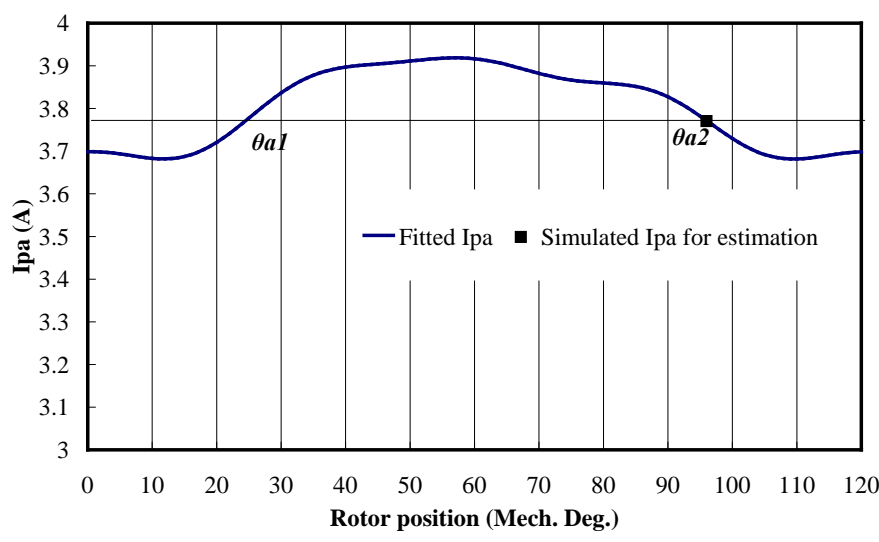

(a) 


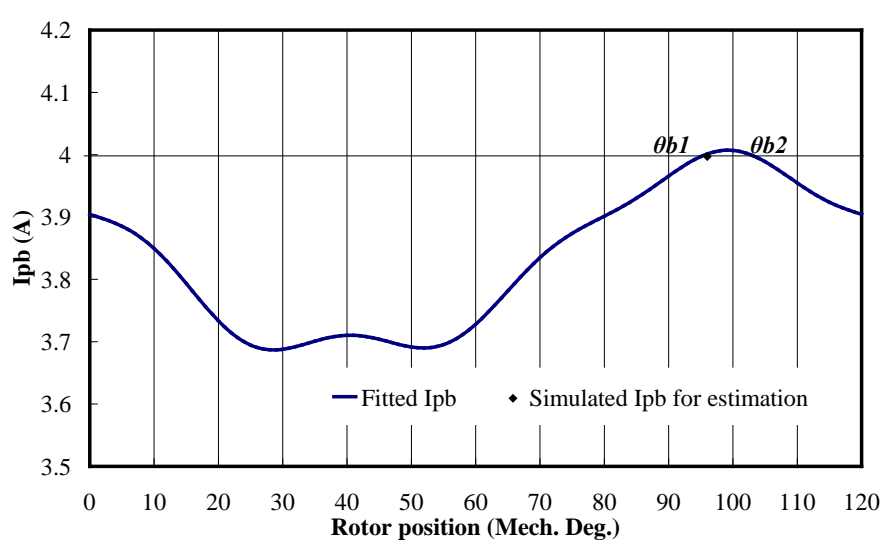

(b)

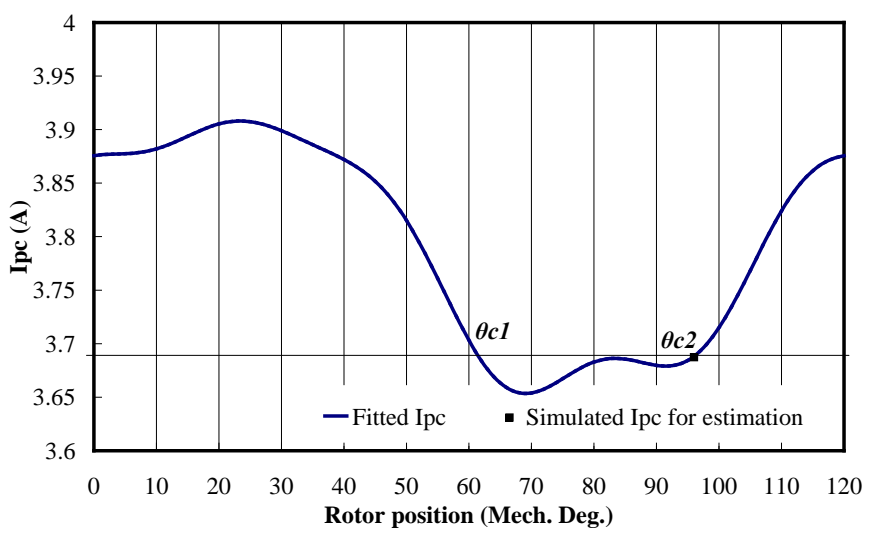

(c)

Figure 6. Estimation of the initial rotor position: (a) phase $a$; (b) phase $b$; and (c) phase $c$.

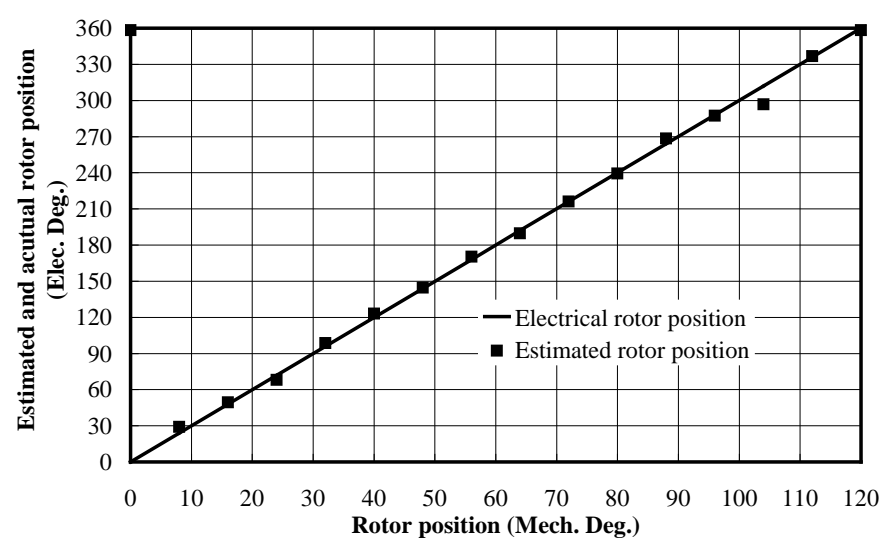

Figure 7. Estimated and actual initial rotor positions.

\section{Estimation Error}

To assess the accuracy of the scheme, an estimation error is defined as

$E r r=\frac{\sqrt{\frac{1}{N} \sum_{i=1}^{N}\left(\theta_{i_{-} \text {est }}-\theta_{i_{-} a c t}\right)^{2}}}{\max \left\{\theta_{i_{-} a c t}\right\}}$ where $N$ is the number of rotor positions, and $\theta_{\text {i_est }}$ and $\theta_{i \_a c t}$ $(i=1,2, \ldots, N)$ are the estimated and actual rotor positions, respectively. The error of the estimation results calculated by (5) is $1.6 \%$, which is satisfactory for general engineering work.

\section{EXPEIRMENTAL VERIFICATION OF INITIAL ROTOR POSITION ESTIMATION}

\section{A. Configuration of Experimental Setup and Measured Peak Currents}

In order to obtain the rotor position related saliency information, several voltage pulses with high amplitudes and short pulsewidth are injected to the stator winding terminals [2$3,5]$. These pulse voltages are produced by PWM inverters in a drive system without additional voltage injection hardware, and the gate signals of the inverters are controlled by a dSPACE DS1104 system. The three phase currents are detected with Hall-effect current probes and are recorded by a digital oscilloscope. The three phase peak current values are used to estimate the initial rotor position. Meanwhile, the actual value of the rotor position is detected from an optical encoder mounted on the shaft of the motor for verifying the estimation.

For the measurement of the peak currents, the peak current value is very sensitive to the applied voltage pulse and resolutions of the current probes, oscilloscope and peripheral devices. To improve the measuring accuracy, the factors determining the peak current values should be investigated. In order to avoid the peak current error due to remnant magnetization and magnetic hysteresis effects, the number of voltage pulses should be increased to diminish the effects. Meanwhile, a series of additional positive and negative voltage pulses should be continuously applied before the peak current is measured.

Besides the magnetic characteristics, the pulsewidth and amplitude of the voltage pulse are also important. To compensate the voltage drop of the PWM inverters during the rapid switching of the inverters, and to protect the inverters, some additional capacitors are used to stabilize the voltage pulses. In the testing system, a fixed sampling time has been determined as a multiple of the duty of the voltage pulses.

With the consideration of the factors mentioned above, totally 36 voltage pulses with 18 positive and 18 negative ones are chosen, and only the current responses to the last four pulses are captured, recorded and stored. The voltage pulse injection experiment as discussed above is conducted for a 6pole SPMSM. At every rotor position, the average values of the four peak currents corresponding to the last four positive or negative voltage pulses are calculated as the final positive or negative peak currents.

\section{B. Initial Rotor Position Estimation Strategy}

The experimental peak currents to positive and negative voltage pulses could be modeled by several periodic functions $[2,5]$. For the peak currents, we have $I_{0 p}=\left(I_{a p}+I_{b p}+I_{c p}\right) / 3$ or $I_{0 n}=\left(\mathrm{I}_{a n}+I_{b n}+I_{c n}\right) / 3$. At a given rotor position, the amplitude of the peak current variation, $\Delta I_{s p}$ and $\Delta I_{s n}$ could be obtained as $\Delta I_{s p}=I_{s p}-I_{O p}$, and $\Delta I_{s n}=I_{s n}-I_{O n}$, where the subscript $s$ represents phases $a, b$ and $c, I_{0 p}$ and $I_{0 n}$ are the average values of the three 
phase positive and negative currents, and $I_{s p}$ and $I_{s n}$ the three phase positive and negative peak currents, respectively. The amplitudes of the three phase positive and negative peak currents are plotted in Fig. 8. With the measured three phase peak current values, the initial rotor position estimation procedure is summarized in Fig. 9. Some of the estimation results are displayed in Fig. 10. The error of the estimation results is $3.4 \%$.

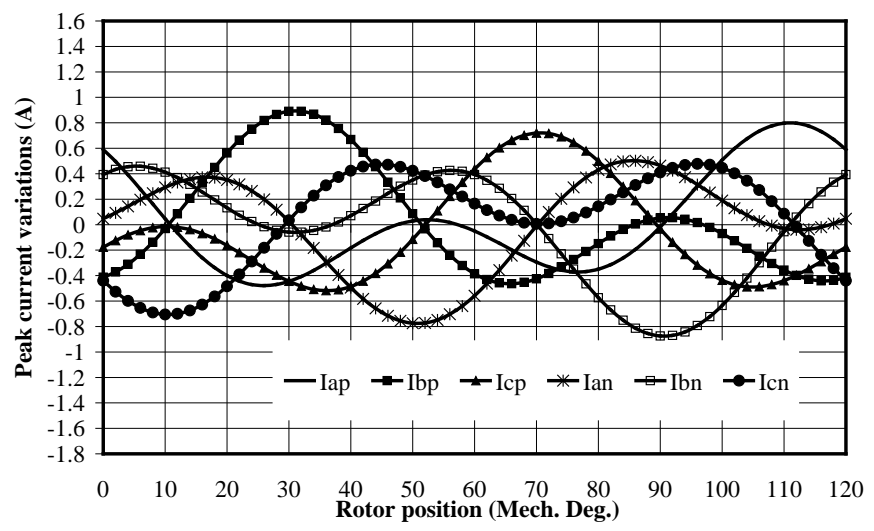

Figure 8. Peak currents versus rotor position.

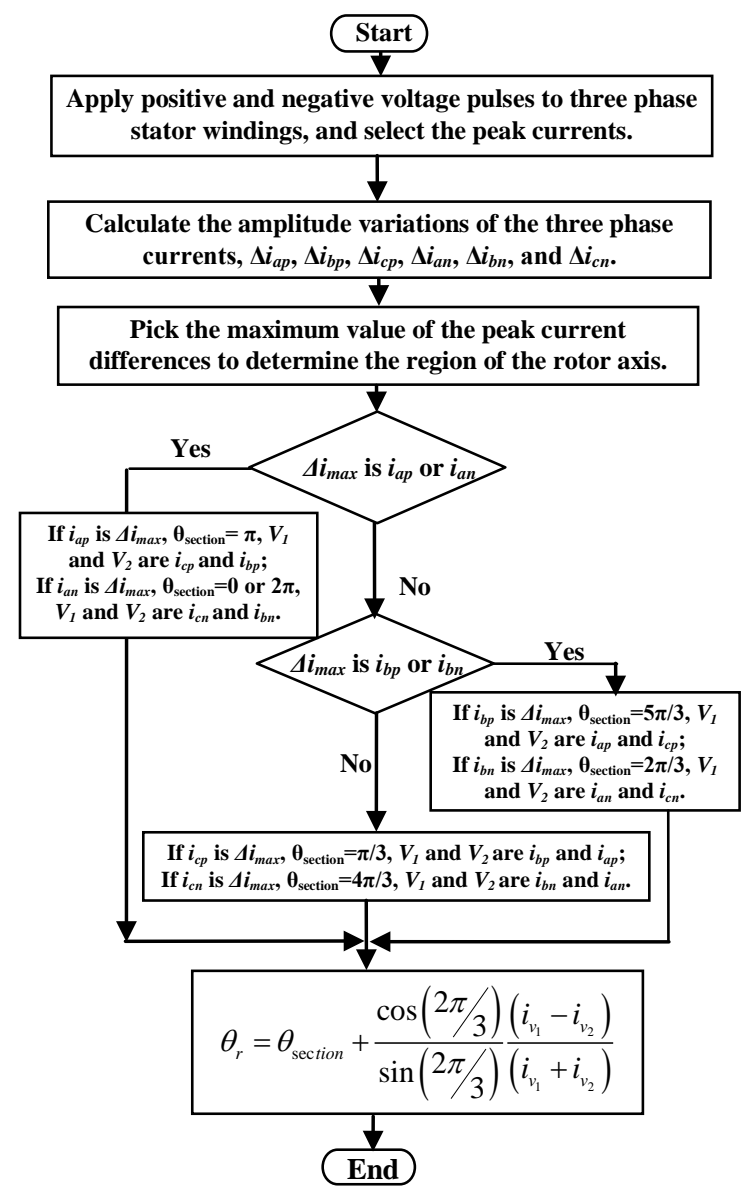

Figure 9. Estimation procedure.

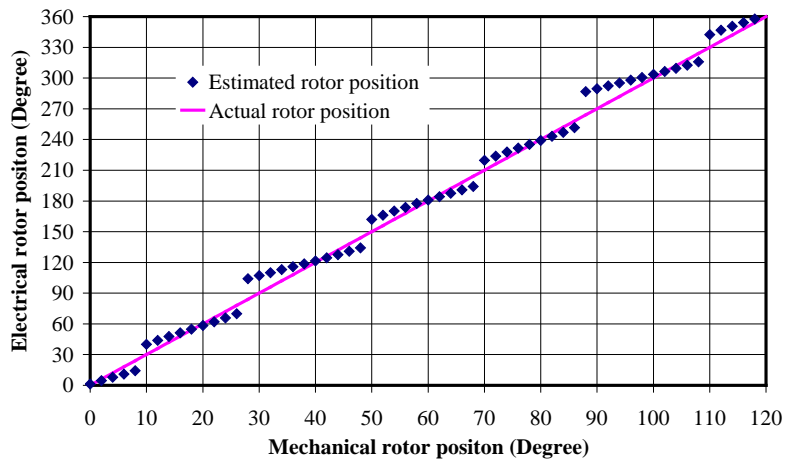

Figure 10. Acutal and estimated initial rotor positions.

\section{IMPLEMENTATION OF A SENSORLESS DTC DRIVE}

With the proposed initial rotor position estimation method, a sensorless DTC scheme is presented and the block diagram of the drive system is plotted in Fig. 11. The parameters of the motor and drive systems have been identified in the previous experiments [4-5]. Based on the designed scheme and the parameters, at an identified initial rotor position, e.g. $\theta=0^{\circ}$, experiments have been conducted. The steady state performance of the experiment with a load torque of $2.5 \mathrm{Nm}$ and a reference speed of $1200 \mathrm{rpm}$ is illustrated in Figs. 12-14. Fig. 12 depicts the rotor speed and electromagnetic torque. The stator flux trajectory and the stator currents are demonstrated in Figs. 13 and 14, respectively.

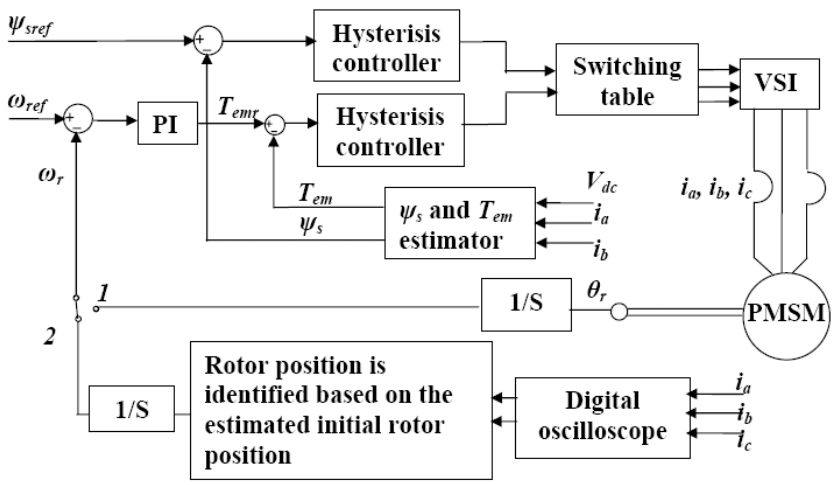

Figure 11. Block diagram of the DTC system with position sensors and the proposed rotor position estimation scheme.

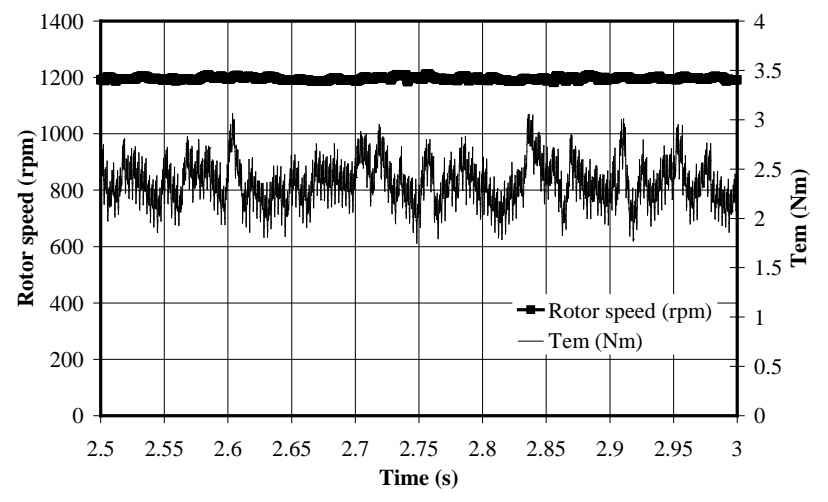

Figure 12. Measured electromagnetic torque and rotor speed. 


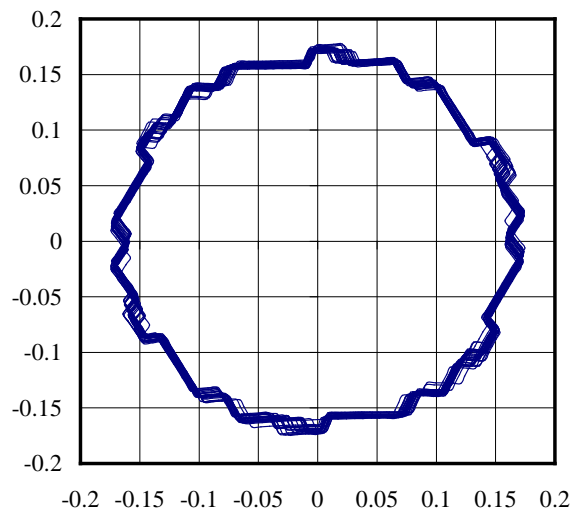

Figure 13. Measured stator flux trajectory.

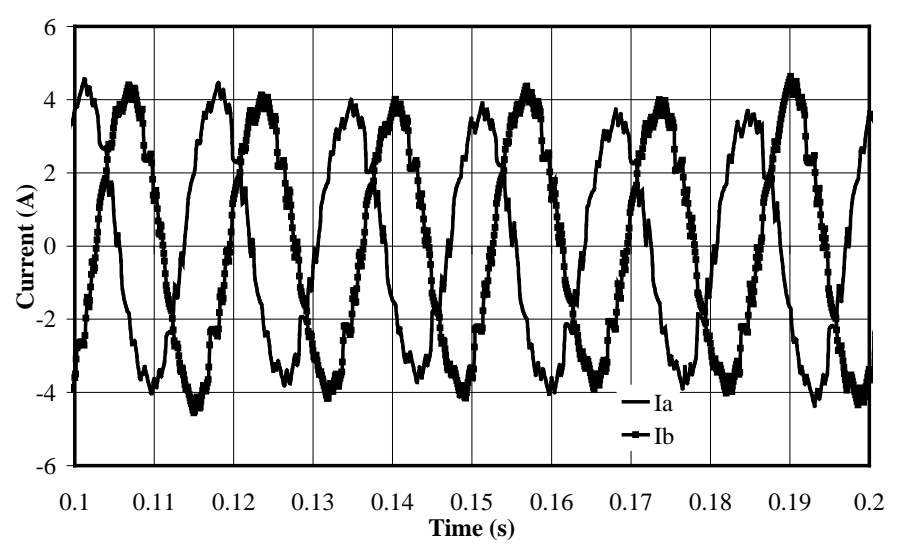

Figure 14. Measured stator currents of phases $a$ and $b$.

\section{CONCLUSIONS}

In this paper, an initial rotor position estimation strategy for DTC PMSM drive is presented. In the scheme, high voltage pulses are applied to amplify the saturation saliencies and corresponding peak currents are used, in combination with the inductance patterns, to determine the $d$-axis position and polarity of the rotor. The scheme can be simulated by using a nonlinear model of PMSM incorporating both structural and saturation saliencies, which are reflected in the winding inductances. The performance of the estimation scheme has been investigated by the simulation results before experimental implementation. The presented strategy has also been implemented in a sensorless DTC scheme for a test SPMSM. Experiments are carried out to confirm the effectiveness of the method, and the performance of the DTC drive system.

\section{REFERENCES}

[1] M. Schroedl, "Sensorless control of ac machines at low speed and standstill based on the "INFORM" method," in Conf. Record $31^{\text {st }}$ IEEEIAS Annual Meeting, vol. 1, Oct. 1996, pp. 270-277.

[2] P. B. Schmidt, M. L. Gasperi, G. Ray, and A. H. Wijenayake, "Initial rotor angle detection of a nonsalient pole permanent magnet synchronous machine," in Conf. Record $32^{\text {nd }}$ IEEE IAS Annual Meeting, Oct. 1997, vol. 1, pp. 459-463.

[3] P. B. Schmidt, "Method and apparatus for rotor angle detection,” U.S. Patent 6172 498, Jan. 9, 2001.

[4] Y. Yan and J. G. Zhu, "Simulation of a direct torque controlled PMSM drive incorporating structural and saturation saliencies," in Proc. $37^{\text {th }}$ IEEE Power Electronics Specialists Conf., June 2006, pp. 1-6.

[5] Y. Yan, J. G. Zhu, Y. G. Guo, and H. W. Lu, "Modeling and simulation of direct torque controlled PMSM drive system incorporating structural and saturation saliencies," in Proc. 41 $1^{\text {st }}$ IEEE IAS Annual Meeting, Tampa, FL, Oct. 2006, pp. 76-83. 